\title{
Analysis of Surface Generation Mechanisms in MOS Capacitors
}

\author{
F. Kong, P. Tanner, L. Hold, J. Han and S. Dimitrijev
}

Abstract - This paper demonstrates the extraction of MOS capacitor minority carrier generation lifetime and surface generation velocity from the measurement of deep-depletion capacitance transient. It is shown that the bulk generation lifetime, the lateral surface generation, and the surface generation under the gate can be separately determined by measuring test structures with different perimeter-to-area ratios.

\section{INTRODUCTION}

The pulsed MOS capacitor transient [1] is the most frequently used method to determine minority carrier generation lifetime and surface generation velocity. Other non-pulse methods such as the linear ramp voltage sweep [2] and steady-state gate-controlled diode [1] are also used. These parameters are important for process monitoring and characterization. This is because they are strongly dependent on densities of crystal defects, heavy metal atoms, stacking faults, dislocations, and interface state densities [1].

In the past, the silicon bulk defects have been gradually eliminated with better crystal growth. However the density of surface defects has not decreased as fast as the bulk defects. Surface effects become increasingly more important during lifetime measurements, because carrier generation can takes place both in the bulk and at the surface.

In this paper, we present experimental measurement of deep-depleted capacitance transients of different kinds of MOS capacitor test structures to identify various generation processes from the semiconductor bulk, lateral spacecharge region surface generation, and surface generation under the gate.

\section{THEORY}

In the commonly used pulsed MOS-C method for lifetime measurement, the MOS capacitor is pulsed from accumulation to deep depletion. Due to thermal generation of the carriers, the non-equilibrium space charge region width after the depleting pulse will eventually collapse to the equilibrium width. The model for the effective generation rate can be written as [1]

$$
\frac{d N_{I}}{d t}=\frac{n_{i}\left(W-W_{f}\right)}{\tau_{g}}+\frac{n_{i} s_{g}^{\prime} A_{s}}{A_{G}}+n_{i} s_{\text {geff }}
$$

F. Kong, P. Tanner, L. Hold, J. Han and S. Dimitrijev are with the School of Microelectronic Engineering, Griffith University, Nathan, Qld 4111, Australia, E-mail: s.dimitrijev @ griffith.edu.au where

$N_{I}$ is the inversion charge per unit area,

$n_{i}$ is the intrinsic carrier concentration,

$\tau_{g}$ is the bulk generation lifetime,

$W$ is the non-equilibrium space charge width,

$W_{f}$ is the final equilibrium scr width,

$s_{g}$ is the lateral surface generation velocity,

$A_{s}$ is the area of the lateral surface charge region,

$A_{G}$ is the gate area,

$s_{\text {geff }}$ is the effective surface generation velocity.

In Eq.(1), the first term accounts for the generation in the bulk, the second term represents the surface generation in the lateral space charge region, and the third term accounts for the scr width independent surface generation under the gate and the quasi-neutral bulk generation related to back-surface generation. The generation components and their locations are shown in Fig. 1.

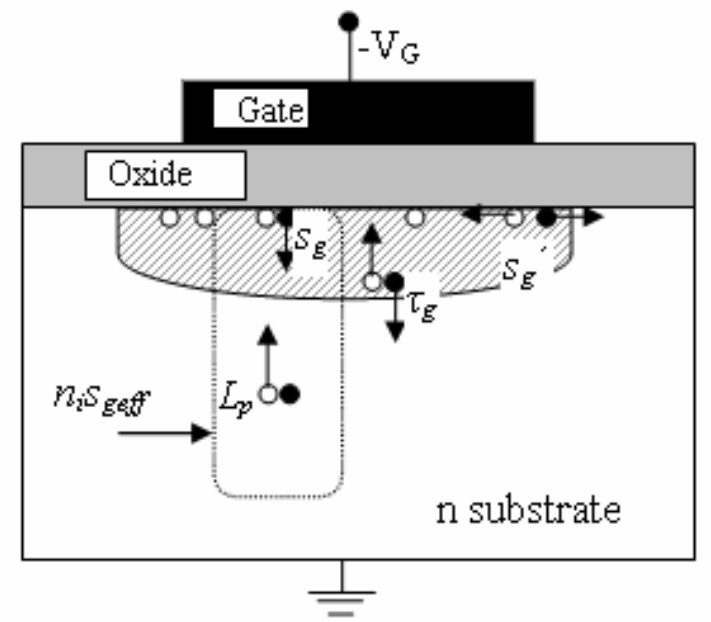

Fig. 1. Generation components in a pulsed MOS capacitor, where $L_{p}$ is the minority carrier diffusion length.

The Zerbst method [3] is widely used for analysis of $\mathrm{C}-\mathrm{t}$ transients. It is based on the following equation:

$$
\begin{aligned}
-\frac{d}{d t}\left(\frac{C_{o x}}{C}\right)^{2}= & \frac{2 n_{i}}{\tau_{g e f f} N_{D}} \frac{\operatorname{Cox}}{C_{f}}\left(\frac{C_{f}}{C}-1\right) \\
& +\frac{K_{o x}}{K_{s}} \frac{2 n_{i} S_{\text {geff }}}{t_{o x} N_{D}}
\end{aligned}
$$

where the effective generation lifetime $\tau_{\text {geff }}$, defined as

$$
\tau_{\text {geff }}=\frac{\tau_{g}}{1+\tau_{g} s_{g}^{\prime} A_{s} /\left[A_{G}\left(W-W_{f}\right)\right]}
$$


is a combination of bulk and lateral surface generation, $C_{o x}$ is the oxide capacitance, $C_{f}$ is the final capacitance, $N_{D}$ is the bulk dopant concentration, $\mathrm{K}_{\mathrm{s}}$ is the silicon dielectric constant, $\mathrm{K}_{\mathrm{ox}}$ is the silicon oxide dielectric constant, and $t_{o x}$ is the oxide thickness. By plotting, $-d\left(C_{o x} / C\right)^{2} / d t$ versus $\left(C_{f} / C-1\right)$, one can extract the effective generation lifetime and the effective surface generation velocity from the slope and the intercept, respectively.

\section{EXPERIMENTAL}

MOS capacitors were fabricated on n-type silicon substrate with doping concentration in the range of 1$1.5 \times 10^{15} \mathrm{~cm}^{-3}$. The bare wafers were first cleaned in a mixture of $\mathrm{H}_{2} \mathrm{SO}_{4}$ and $\mathrm{H}_{2} \mathrm{O}_{2}$, followed by an RCA clean. This was then followed by $1 \% \mathrm{HF}$ dip for $1 \mathrm{~min}$. The gate oxide was grown in dry $\mathrm{O}_{2}$ at $880^{\circ} \mathrm{C}$. After that aluminum was thermally evaporated to form the gate electrodes. Square and finger capacitors with area of $0.0025 \mathrm{~cm}^{2}$ and $0.002 \mathrm{~cm}^{2}$ respectively were then defined by photolithography.

The MOS capacitors were characterized by highfrequency capacitance-voltage (HFCV) and capacitancetime (C-t) measurements at room temperature. The HFCV measurements were performed at the sweep rate of $0.1 \mathrm{~V} / \mathrm{s}$ and the sweep range from $2 \mathrm{~V}$ to $-6 \mathrm{~V}$, using a computercontrolled HP4284A LCR meter. The gate-oxide thickness, determined from the accumulation capacitance, was 18.6 $\mathrm{nm}$.

During the C-t measurements, the return from nonequilibrium into equilibrium strong inversion state was recorded as the capacitance transient. Zerbst plots of the C$\mathrm{t}$ responses were used to extract the minority carrier lifetime and surface generation velocity.

\section{RESULTS AND DISCUSSION}

The HFCV curves for square and finger test structures are shown in Fig 2. There is no significant difference between the two curves except in the magnitude of the capacitance in accumulation due to difference in the capacitor areas.

The C-t response curve for finger and square MOS capacitor structures is shown in Fig 3. A very different behavior is observed for the finger test structure when compared to the square test structure. The response of the $\mathrm{C}$ - $\mathrm{t}$ for the finger structure is a lot faster than for the square structure, although the capacitance in both cases saturates at the strong-inversion level. Note that the initial biasing of the gates at inversion, instead of at accumulation, the surface generation under the gate is minimized. We believe the short $\mathrm{C}$ - $\mathrm{t}$ response of the finger MOS capacitor is primarily due to the lateral scr surface generation.

The corresponding Zerbst plots of the C-t responses for the square and finger structures are shown in Fig 4. The Zerbst plot is highly non-linear for the finger test structure. Such a curve is shown in Fig 4 (Curve (b)). In contrast, the curve (a) derived from the C-t response of the square sample has a much wider linear portion.

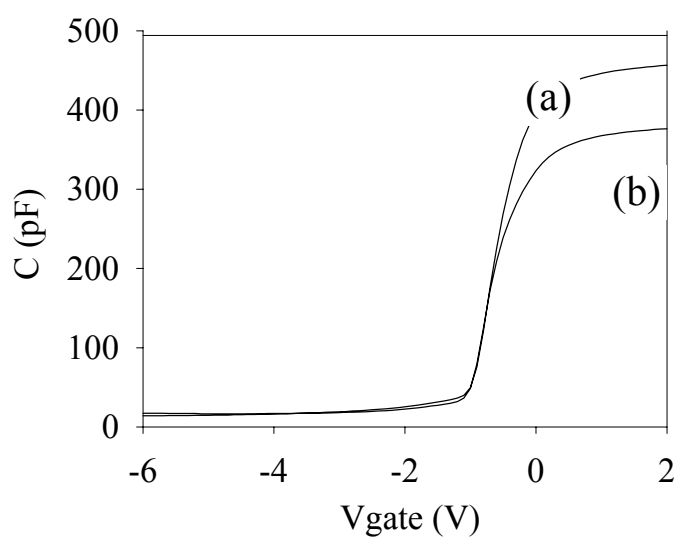

Fig. 2. High frequency C-V measurements for (a) square and (b) finger MOS capacitors, respectively.

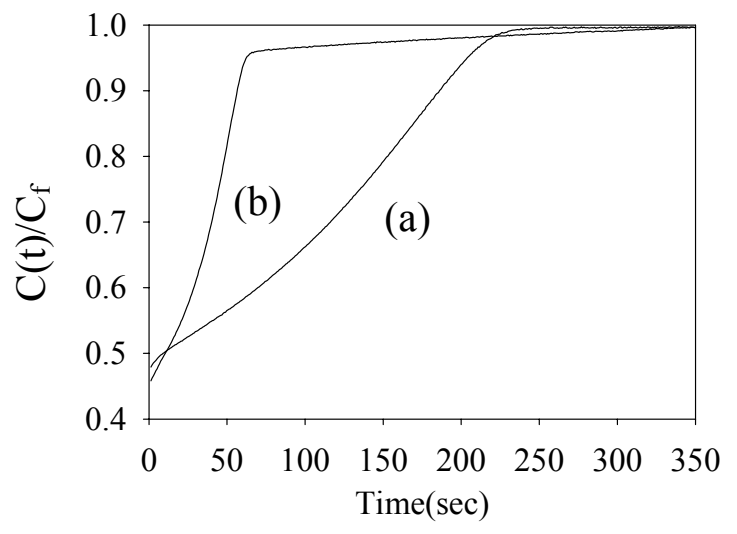

Fig. 3. C-t response curves for (a) square and (b) finger test structures. Both C-t were obtained by pulsing from $-2 \mathrm{~V}$ to $-5 \mathrm{~V}$ (i.e. from inversion to strong inversion).

The effective generation lifetimes and surface generation velocities obtained from the Zerbst plots for the two different samples are listed in Table 1.

TABLE I

EXTRACTED EFFECTIVE GENERATION LIFETIME AND SURFACE GENERATION VELOCITY FROM ZERBST PLOTS OF FIG 3.

\begin{tabular}{|l|l|l|l|l|}
\hline & $\begin{array}{l}\text { Area } \\
\left(\mathrm{cm}^{2}\right)\end{array}$ & $\begin{array}{l}\text { Perimeter } \\
(\mu \mathrm{m})\end{array}$ & $\begin{array}{l}\tau_{\text {geff }} \\
(\mu \mathrm{s})\end{array}$ & $\begin{array}{l}s_{\text {geff }} \\
(\mathrm{cm} / \mathrm{s})\end{array}$ \\
\hline Square & 0.0025 & 2000 & 60.0 & 1.12 \\
\hline Finger & 0.002 & 4440 & 26.3 & 4.83 \\
\hline
\end{tabular}


The extracted effective minority generation lifetime for the finger structure is reduced more than twice in comparison to the square structure. This can be explained by the fact that the finger structure has a higher perimeter to area ratio than the square structure. However, the extracted effective surface generation velocity is also increased four times for the finger structure. This was not expected because the gate oxides of the both capacitors were grown under the same conditions and physically close to each other. Possible explanation could be that a part of the lateral surface generation does not change linearly with $W$ and is therefore contributing to the value of $s_{\text {geff. A }}$ possible reason for the lateral surface generation not changing linearly with $W$ could be surface ions on the oxide surrounding the gate, which can significantly influence the lateral surface generation [4].

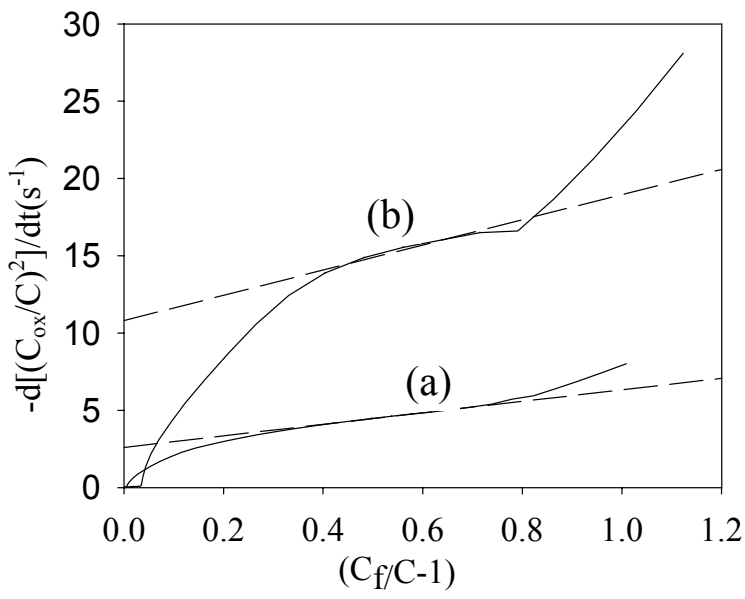

Fig. 4. Zerbst plots for (a) square and (b) finger test structures.

By assuming the first term of Eq. (1) is the same for both the finger and the square structures, one can solve two simultaneous equations to get $\tau_{g}=228.97 \mu$ s and $s_{g}{ }^{\prime}=$ $153.48 \mathrm{~cm} / \mathrm{s}$ using the extracted $\tau_{\text {geff }}$ from Table 1 . Hence, it is possible to obtain the individual components of the generation rates as shown in Table 2 , where $G_{1}, G_{2}$, and $G_{3}$ are the first, second, and third terms in the right hand side of Eq. (1), respectively.

TABLE II

DIFFERENT COMPONENTS OF GENERATION RATES AT ROOM TEMPERATURE.

\begin{tabular}{|l|l|l|l|}
\hline & $G_{1}\left(\mathrm{~cm}^{-2} \mathrm{~s}^{-1}\right)$ & $G_{2}\left(\mathrm{~cm}^{-2} \mathrm{~s}^{-1}\right)$ & $G_{3}\left(\mathrm{~cm}^{-2} \mathrm{~s}^{-1}\right)$ \\
\hline Square & $4.81 \times 10^{9}$ & $1.35 \times 10^{10}$ & $1.19 \times 10^{10}$ \\
\hline Finger & $4.81 \times 10^{9}$ & $3.75 \times 10^{10}$ & $4.91 \times 10^{10}$ \\
\hline
\end{tabular}

It can be seen that both the lateral surface generation and the generation under the gate are much higher than the bulk generation for both test structures.

\section{CONCLUSION}

We have used the C-t transients of MOS capacitors to analyze different generation mechanisms in silicon. It is demonstrated that using different structures we can separate out different generation components. The lateral surface generation can be important and needs to be taken into account for interpretation of MOS capacitance transient measurements.

\section{REFERENCES}

[1] D. K. Schroder, Semiconductor Material and Device Characterisation, ch.7. New York: Wiley, 1998.

[2] R. F. Pierret, "A linear sweep MOS-C technique for determining minority carrier lifetimes," IEEE Trans. Electron Devices, vol.19, p.869-873, July 1972.

[3] M. Zerbst, "Relaxation effects at semiconductor-insulator interfaces," (in German), Z. Angew. Phys. 22, 30-33, May 1966.

[4] R. F. Pierret and D. W. Small, "Effects of lateral surface generation on the MOS-C linear-sweep and C-t transient characteristics," IEEE Trans. Electron Devices, vol.20, p.457-458, April 1973. 